\title{
A Nonredundant Near-Field to Far-Field Transformation with Spherical Spiral Scanning for Nonspherical Antennas
}

\author{
F. D’Agostino, F. Ferrara, C. Gennarelli ${ }^{*}$, R. Guerriero, M. Migliozzi and G. Riccio
}

D.I.I.I.E - University of Salerno, via Ponte Don Melillo, 84084 Fisciano (Salerno), Italy

\begin{abstract}
A fast and accurate near-field - far-field transformation technique with spherical spiral scanning tailored for nonspherical antennas, i.e., radiating systems with two dimensions very different from the third one, is developed in this paper. To this end, an antenna with one or two predominant dimensions (e.g., base station antennas and reflectarrays) is no longer considered as enclosed in a sphere, but in a prolate or oblate ellipsoid, respectively, thus allowing a remarkable reduction of the number of the near-field data to be acquired. Moreover, these source modellings remain quite general and contain the spherical one as particular case. Some numerical simulations, assessing the accuracy of the far-field reconstruction process and its robustness with respect to random errors affecting the data, are reported.
\end{abstract}

\section{INTRODUCTION}

As it is well-known, near-field - far-field (NF-FF) transformation techniques play a significant role in modern antenna measurements [1-3]. As a matter of fact, the pattern evaluation from NF measurements allows one to overcome those drawbacks which, for electrically large radiating systems, make unpractical to measure the pattern in a conventional FF range. As suggested in [4], by employing continuous and synchronized movements of the positioning systems of the probe and antenna under test (AUT), the time required for the acquisition of the NF data can be drastically reduced. By following this suggestion, innovative NF-FF transformation techniques from a nonredundant number of data collected along spirals wrapping the conventional scanning surfaces have been recently developed [5-7]. In particular, a $\mathrm{NF}-\mathrm{FF}$ transformation with helicoidal scanning has been proposed in [5], a planar spiral arrangement of samples has been considered in [6], and a NF-FF transformation with spherical spiral scanning has been developed in [7]. In all the cases, a nonredundant representation of the voltage data acquired by the measurement probe on the considered scanning curve has been developed by applying the theoretical results on the nonredundant sampling representations of electromagnetic (EM) fields [8] and assuming the AUT enclosed in a sphere. This, in addition to the choice of the curve step equal to the sample spacing needed to interpolate the data along the corresponding meridian curve (generatrix, radial line, and meridian), has allowed one to obtain the required two-dimensional optimal sampling interpolation (OSI) formula. It has been so possible to recover the NF data needed by the NF-FF transformations employing the corresponding conventional scannings [3]. At last, a unified theory of the spiral scannings has been provided in [9]. As a matter of fact, it has been proved that the voltage acquired by a nondirective probe can be reconstructed on a quite arbitrary rotational surface from a nonredundant number of its samples

*Address correspondence to this author at the D.I.I.I.E - University of Salerno, via Ponte Don Melillo, 84084 Fisciano (Salerno), Italy;

E-mail: gennar@diiie.unisa.it lying on a proper spiral wrapping the surface. The only required condition is that such a surface is obtained by rotating a meridian curve always external to the cone having the vertex at the observation point and tangent to the AUT sphere. The proof has been attained by revisiting the approach in [8] for obtaining the optimal phase function to extract from the voltage expression and the parameter to be used for representing the scanning curve. It is worth noting that the voltage can be reconstructed by using the same interpolation scheme, even if the spiral lies on geometrically different surfaces.

Unfortunately, when considering elongated or quasiplanar antennas having two dimensions very different from the third one, the spherical AUT modelling, even if quite general, induces a "volumetrical" redundancy, which implies an unnecessary increase in the number of required data.

The goal of this paper is just to overcome the above drawback by developing fast and accurate NF-FF transformation techniques with spherical spiral scanning tailored for these radiating systems. To this end, an antenna with one or two predominant dimensions is no longer considered as enclosed in a sphere, but in a prolate (Fig. 1) or oblate (Fig. 2) ellipsoid, respectively, thus remarkably reducing the number

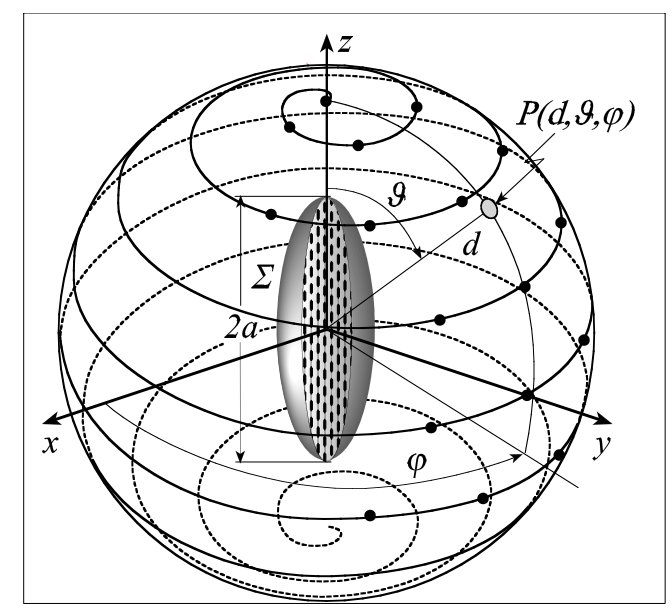

Fig. (1). Geometry of the problem for elongated antennas. 
of required data. Moreover, these source modellings remain quite general and contain the spherical one as particular case. For sake of simplicity, the use of an ideal probe is assumed in the following, the extension to the case of a real probe being straightforward.

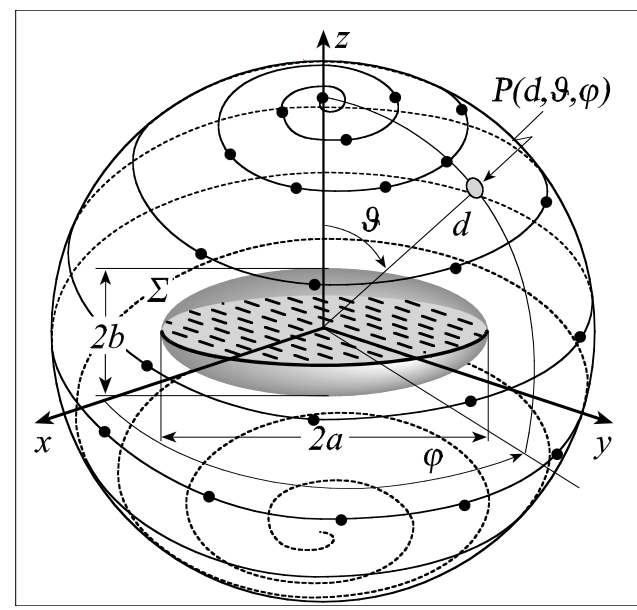

Fig. (2). Geometry of the problem for quasi-planar antennas.

\section{THE SPHERICAL AUT MODELLING CASE}

For reader's convenience, the main results concerning the spherical spiral scanning when using a spherical AUT modelling are summarized in this section.

Let us observe the field radiated by an AUT on a sphere of radius $d$ in the NF region and adopt the spherical coordinate system $(r, \vartheta, \varphi)$ to denote the point $P$ (see Figs. 1 and 2). According to [8], if the AUT is modelled as enclosed in the smallest sphere of radius $a$ able to contain it and the spiral is described by means of a proper analytical parameterization $\underline{r}=\underline{r}(\xi)$, the "reduced electric field"

$\underline{F}(\xi)=\underline{E}(\underline{r}(\xi)) \mathrm{e}^{\mathrm{j} \gamma(\xi)}$,

$\gamma(\xi)$ being a phase function to be determined, can be closely approximated by a spatially bandlimited function. The corresponding bandlimitation error becomes negligible as the bandwidth exceeds a critical value $W_{\xi}$ [8], so that it can be effectively controlled by choosing a bandwidth equal to $\chi^{\prime} W_{\xi}, \chi^{\prime}>1$ being an excess bandwidth factor.

According to [8], a nonredundant sampling representation of the EM field on a spherical spiral with constant angular step $\Delta \vartheta$ can be obtained by using the following expressions for the optimal parameterization $\xi$ and phase function $\gamma:$

$\xi=\xi(s)=\frac{\beta}{2 W_{\xi}} \int_{0}^{s}\left[\max _{\underline{r}^{\prime}} \hat{R} \cdot \hat{t}-\min _{\underline{r}^{\prime}} \hat{R} \cdot \hat{t}\right] \mathrm{d} s^{\prime}$

$\gamma(s)=\frac{\beta}{2} \int_{0}^{s}\left[\max _{\underline{r}^{\prime}} \hat{R} \cdot \hat{t}+\min _{\underline{r}^{\prime}} \hat{R} \cdot \hat{t}\right] \mathrm{d} s^{\prime}$ where $\underline{r}^{\prime}$ denotes the source point, $s$ is the arclength of the spiral, $\hat{t}$ is the unit vector tangent to it at the considered point $Q$ on the spiral, $\beta$ is the wavenumber and $\hat{R}$ is the unit vector from the source point to $Q$. The coordinates of $Q$ are given by:

$$
\left\{\begin{array}{l}
x=d \sin \theta \cos \phi \\
y=d \sin \theta \sin \phi \\
z=d \cos \theta
\end{array}\right.
$$

wherein $\phi$ is the parameter describing the spiral. Note that the spiral angle $\theta=k \phi$, unlike the zenithal angle $\vartheta$, can assume negative values. Moreover, the spiral angle $\phi$ is always continuous, whereas, according to (4), the azimuthal angle $\varphi$ exhibits a discontinuity jump of $\pi$ when the spiral crosses the poles. Such a curve can be obtained by radially projecting on the measurement sphere a proper spiral wrapping the AUT sphere. In order to allow the two-dimensional interpolation, the angular step of the spiral must be chosen equal to the sample spacing required to interpolate the field along a meridian $[8,9]$. As a consequence, the parameter $k$ is such that the angular step, determined by the consecutive intersections $Q(\phi)$ and $Q(\phi+2 \pi)$ of the spiral with the considered meridian, is $\Delta \theta=\Delta \vartheta=2 \pi /\left(2 N^{\prime \prime}+1\right)$, with $N^{\prime \prime}=\operatorname{Int}\left(\chi N^{\prime}\right)+1$ and $N^{\prime}=\operatorname{Int}\left(\chi^{\prime} \beta a\right)+1$. Accordingly, being $\Delta \theta=2 \pi k$, it follows that $k=1 /\left(2 N^{\prime \prime}+1\right)$. The function $\operatorname{Int}(x)$ gives the integer part of $x$, and $\chi>1$ is an oversampling factor.

The extreme values of $\hat{R} \cdot \hat{t}$ in (2) and (3) are determined $[7,9]$ by considering the intersection of the plane defined by $\hat{t}$ and the unit vector $\hat{r}$ (pointing from the origin to $Q$ ) with the cone having the vertex at $Q$ and the generatrices coincident with the tangents to the AUT ball. As shown in [9], it results:

$$
\begin{aligned}
& \gamma=\beta \int_{0}^{d} \sqrt{1-a^{2} / r^{\prime 2}} \mathrm{~d} r^{\prime}=\beta \sqrt{d^{2}-a^{2}}-\beta a \cos ^{-1}\left(\frac{a}{d}\right) \\
& \xi=\frac{\beta a}{W_{\xi}} \int_{0}^{\phi} \sqrt{k^{2}+\sin ^{2} k \phi^{\prime}} \mathrm{d} \phi^{\prime}
\end{aligned}
$$

As can be seen, $\xi$ is $\beta / W_{\xi}$ times the curvilinear abscissa along the spiral wrapping the sphere modelling the AUT. Since such a spiral is a closed curve, it is convenient to choose the bandwidth $W_{\xi}$ such that the angular like parameter $\xi$ covers a $2 \pi$ range when the whole projecting curve is described. Therefore,

$W_{\xi}=\frac{\beta a}{\pi} \int_{0}^{(2 N "+1) \pi} \sqrt{k^{2}+\sin ^{2} k \phi^{\prime}} \mathrm{d} \phi^{\prime}$

By taking into account the above representation, the OSI formula for reconstructing the reduced field at any point $Q$ of the spiral is $[7,9]$ : 
$\underline{F}(\xi)=\sum_{m=m_{0}-p+1}^{m_{0}+p} \underline{F}\left(\xi_{m}\right) \Omega_{M}\left(\xi-\xi_{m}\right) D_{M^{n}}\left(\xi-\xi_{m}\right)$

where $2 p$ is the number of retained samples $\underline{F}\left(\xi_{m}\right)$, $m_{0}=\operatorname{Int}(\xi / \Delta \xi)$ is the index of the sample nearest (on the left) to $Q$, and

$\xi_{m}=m \Delta \xi=2 \pi m /(2 M "+1)$

with $M^{\prime \prime}=\operatorname{Int}\left(\chi M^{\prime}\right)+1$ and $M^{\prime}=\operatorname{Int}\left(\chi^{\prime} W_{\xi}\right)+1$. Moreover,

$D_{M "}(\xi)=\frac{\sin \left(\left(2 M^{\prime \prime}+1\right) \xi / 2\right)}{\left(2 M^{\prime \prime}+1\right) \sin (\xi / 2)}$

$\Omega_{M}(\xi)=\frac{T_{M}\left[-1+2(\cos (\xi / 2) / \cos (\bar{\xi} / 2))^{2}\right]}{T_{M}\left[-1+2 / \cos ^{2}(\bar{\xi} / 2)\right]}$

are the Dirichlet and Tschebyscheff Sampling functions, wherein $T_{M}(\xi)$ is the Tschebyscheff polynomial of degree $M=M^{\prime \prime}-M^{\prime}$ and $\bar{\xi}=p \Delta \xi$. It is worthy to note that, when interpolating the field in the neighbourhood of the poles $(\vartheta=0$ and $\vartheta=\pi$ ), it is necessary to increase the excess bandwidth factor $\chi^{\prime}$ in order to avoid a significant growth of the bandlimitation error. This is mainly due to the fact that small variations of $\xi$ correspond to very large changes of $\phi$ in these zones.

The OSI formula (8) can be applied to determine the "intermediate samples", i.e., the reduced field values at the intersection points between the spiral and the meridian passing through $P$. Once these last have been evaluated, an analogous expansion along $\vartheta$ can be used to reconstruct the field at $P$ [9]. It is so possible to get the NF data needed to perform the spherical NF-FF transformation [10], as modified in [11].

\section{EXTENSION TO NONSPHERICAL ANTENNAS}

The aim of this section is just the extension of the previously described sampling representation to the case of nonspherical antennas, i.e., those having one or two predominant dimensions. When dealing with such a kind of antennas, it is no longer convenient to adopt the smallest sphere as surface enclosing them but, as suggested in [8], a proper rotational surface $\Sigma$ bounding a convex domain.

The parameterization $\eta$ to be used for describing a meridian and the corresponding phase function $\psi$ can be derived by taking into account that the extreme values of the inner product in (2) and (3) occur (see Fig. 3) at the two tangency points $P_{1,2}$ on $C^{\prime}$ (intersection curve between the meridian plane $\Pi$ and 2 ). Accordingly, by choosing $W_{\eta}=\beta \ell^{\prime} / 2 \pi$ ( $\ell^{\prime}$ being the length of $C^{\prime}$ ), it results [8]:

$\eta=\frac{\pi}{\ell^{\prime}}\left[R_{1}-R_{2}+s_{1}^{\prime}+s_{2}^{\prime}\right]$
$\psi=\frac{\beta}{2}\left[R_{1}+R_{2}+s_{1}^{\prime}-s_{2}^{\prime}\right]$

where $s_{1,2}^{\prime}$ are the arclength coordinates of $P_{1,2}$ and $R_{1,2}$ the distances from $P$ to $P_{1,2}$ (Fig. 3).

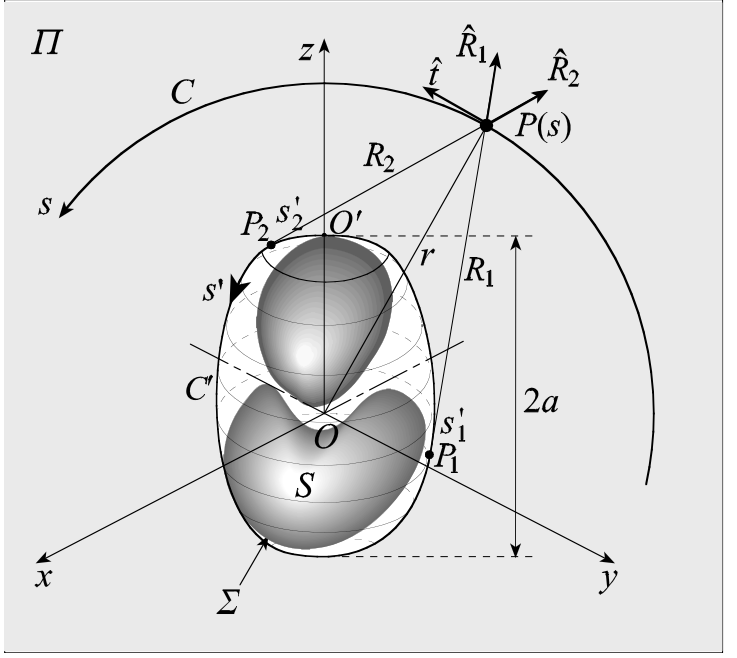

Fig. (3). Relevant to an observation meridian.

Some intriguing questions now arise. What are the equations of the spiral and the optimal expressions of $\xi$ and $\gamma$, which allow to obtain a nonredundant representation along such a curve?

According to a heuristic reasoning, by paralleling the procedure of the previous section, the spiral can be obtained by projecting on the observation surface a proper spiral that wraps around the surface $\Sigma$ modelling the AUT. The step of such a spiral is equal to the sample spacing $\Delta \eta=2 \pi /\left(2 N^{\prime \prime}+1\right)$ needed to interpolate the field along a meridian. It is worthy to note that, as before, $N^{\prime \prime}=\operatorname{Int}\left(\chi N^{\prime}\right)+1$, but now $N^{\prime}=\operatorname{Int}\left(\chi^{\prime} W_{\eta}\right)+1$. The projec-

tion is obtained via the curves at $\eta=$ const that, in such a case, take the role of the radial lines of the spherical modelling. Accordingly, the parametric equations of the spiral become:

$\left\{\begin{array}{l}x=d \sin \theta(\eta) \cos \phi \\ y=d \sin \theta(\eta) \sin \phi \\ z=d \cos \theta(\eta)\end{array}\right.$

wherein $\eta=k \phi=\phi /\left(2 N^{\prime \prime}+1\right)$.

Again a heuristic reasoning allows the determination of the parameter $\xi$ and phase factor $\gamma$ for obtaining a nonredundant sampling representation along the spiral. In particular, by generalizing the corresponding relations for the case of spherical modelling (see (5) and (6)), $\gamma$ coincides with the phase function $\psi$ relevant to a meridian, and $\xi$ is $\beta / W_{\xi}$ times the arclength of the projecting point that lies on the spiral wrapping the surface $\Sigma$. Moreover, $W_{\xi}$ is chosen equal 
to $\beta / \pi$ times the length of the spiral wrapping the surface $\Sigma$ from pole to pole. Namely, the spiral, $\gamma$ and $\xi$ are such that they coincide with those relevant to the spherical modelling, when $\Sigma$ leads to a sphere.

The OSI expansion (8) can be still employed to recover the intermediate samples. Once these samples have been evaluated, the field at any point $P$ on the observation sphere can be reconstructed via the following OSI expansion:

$\underline{F}(\eta(\vartheta), \varphi)=\sum_{n=n_{0}-q+1}^{n_{0}+q} \underline{F}\left(\eta_{n}\right) \Omega_{N}\left(\eta-\eta_{n}\right) D_{N^{\prime \prime}}\left(\eta-\eta_{n}\right)$

where $\left.N=N^{\prime \prime}-N^{\prime}, \quad n_{0}=\operatorname{Int}\left[\left(\eta-\eta_{0}\right) / \Delta \eta\right)\right], 2 q$ is the number of the retained intermediate samples $\underline{F}\left(\eta_{n}\right)$,

$\eta_{n}=\eta_{n}(\varphi)=k \varphi+n \Delta \eta=\eta_{0}+n \Delta \eta$

and all the other symbols have the same or analogous meaning as in (8).

Let us now briefly discuss the reasons for applying a heuristic approach. First of all, the rigorous analytical determination of the optimal spiral and the evaluation of the corresponding phase factor and parameterization become much more difficult or impossible, since some analytical considerations are no longer valid when adopting a modelling different from the spherical one. On the other hand, the heuristic approach gives an easy to handle tool for determining them. Moreover, it can be easily applied to different AUT modellings, as those proposed in $[12,13]$ with reference to the plane-polar and cylindrical scanning case, respectively.

\section{ELLIPSOIDAL AUT MODELLING CASES}

An effective modelling for an elongated AUT is obtained by considering it as enclosed in the smallest prolate ellipsoid having major and minor semi-axes equal to $a$ and $b$ (see Figs. 1 and 4), whereas an appropriate modelling for a quasiplanar source is got by enclosing it in an oblate ellipsoid (Figs. 2 and 5). In these cases, the optimal expressions for the bandwidth $W_{\eta}$, the phase factor $\psi$ and parameterization $\eta$ relevant to a meridian become [8]:

$W_{\eta}=\frac{4 a}{\lambda} E\left(\pi / 2 \mid \varepsilon^{2}\right)$

$\psi=\beta a\left[v \sqrt{\frac{v^{2}-1}{v^{2}-\varepsilon^{2}}}-E\left(\cos ^{-1} \sqrt{\frac{1-\varepsilon^{2}}{v^{2}-\varepsilon^{2}}} \mid \varepsilon^{2}\right)\right]$

$\eta=\frac{\pi}{2}\left\{\begin{array}{cc}\mathrm{E}\left(\sin ^{-1} u \mid \varepsilon^{2}\right) / \mathrm{E}\left(\pi / 2 \mid \varepsilon^{2}\right)+1 & \text { prolate } \\ \mathrm{E}\left(\sin ^{-1} u \mid \varepsilon^{2}\right) / \mathrm{E}\left(\pi / 2 \mid \varepsilon^{2}\right) & \text { oblate }\end{array}\right.$

where $\lambda$ is the wavelength, $u=\left(r_{1}-r_{2}\right) / 2 f$ and $v=\left(r_{1}+r_{2}\right) / 2 a$ are the elliptic coordinates, $r_{1,2}$ being the distances from $P$ to the foci and $2 f$ the focal distance of $C^{\prime}$.

Moreover, $\varepsilon=f / a$ is the eccentricity of $C^{\prime}$ and $E(\cdot \mid \cdot)$ de- notes the elliptic integral of second kind. It is worth noting that, in any meridian plane, the curves $\psi=$ const and $\eta=$ const (see Figs. 4, 5) are ellipses and hyperbolas confocal to $C^{\prime}$, instead of circumferences and radial lines of the spherical source modelling.

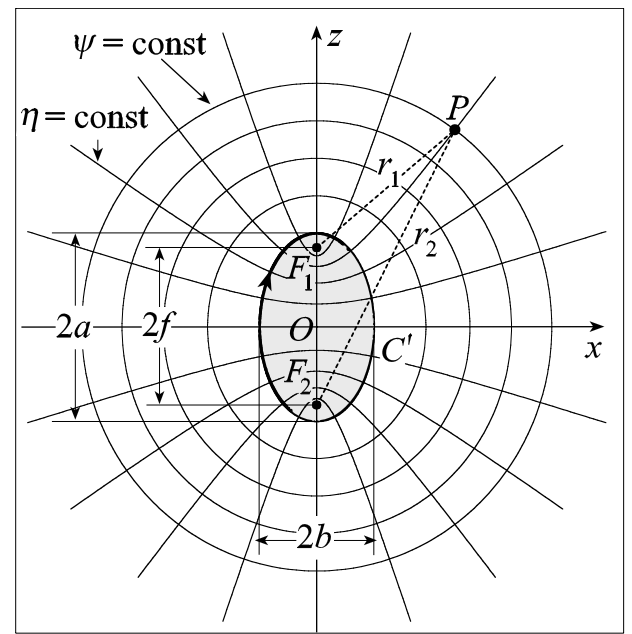

Fig. (4). Ellipsoidal source modelling: prolate case.

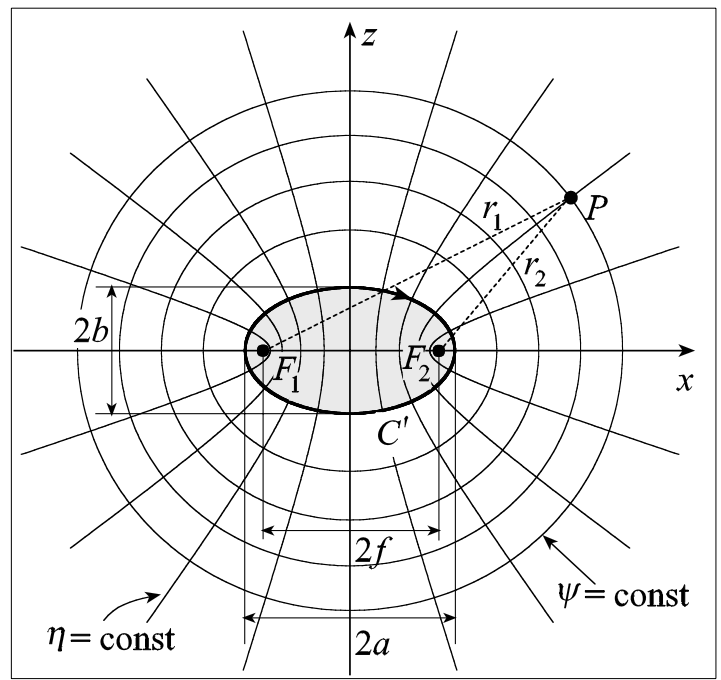

Fig. (5). Ellipsoidal source modelling: oblate case.

\section{NF-FF TRANSFORMATION WITH SPHERICAL SCANNING}

The key steps of the classical NF-FF transformation with spherical scanning [10] as modified in [11] are reported in this section.

According to [10], the tangential electric field in the FF region can be expressed in terms of the following truncated spherical wave expansion:

$\underline{E}_{t}(R \rightarrow \infty, \Theta, \Phi)=\frac{\mathrm{e}^{-\mathrm{j} \beta R}}{R}$.

$\cdot \sum_{n=1}^{N_{\max }} \sum_{k=-n}^{n}\left[\mathrm{j}^{n+1} A_{1_{n k}} \underline{f}_{1_{n k}}(\Theta)+\mathrm{j}^{n} A_{2_{n k}} \underline{f}_{2_{n k}}(\Theta)\right] \mathrm{e}^{\mathrm{j} k \Phi}$ 
where the spherical coordinate system $(R, \Theta, \Phi)$ has been adopted to specify the FF observation point.

The choice of the highest spherical wave to be considered is determined in the classical approach according to the following rule-of-thumb:

$N_{\max }=\operatorname{Int}(\beta a)+10$

where $a$ is the radius of the smallest sphere enclosing the AUT. Whereas in the approach [11], the highest spherical harmonic is rigorously fixed by the aforementioned bandlimitation properties of the radiated EM fields [8]. Accordingly:

$N_{\max }=\operatorname{Int}\left(\chi^{\prime} \beta a\right)+1$

The vectorial functions $\underline{f}_{1,2_{n k}}(\Theta)$ are given by:

$\underline{f}_{1_{n k}}(\Theta)=\left(\frac{-k}{|k|}\right)^{k} \frac{1}{\sqrt{2 \pi n(n+1)}}$

$\cdot\left[\frac{\mathrm{j} k}{\sin \Theta} \bar{P}_{n}^{|k|}(\cos \Theta) \hat{\Theta}+\frac{\mathrm{d}}{\mathrm{d} \Theta} \bar{P}_{n}^{|k|}(\cos \Theta) \hat{\Phi}\right]$

$\underline{f}_{2 n k}(\Theta)=\left(\frac{-k}{|k|}\right)^{k} \frac{1}{\sqrt{2 \pi n(n+1)}}$.

$\cdot\left[\frac{\mathrm{d}}{\mathrm{d} \Theta} \bar{P}_{n}^{|k|}(\cos \Theta) \hat{\Theta}+\frac{\mathrm{j} k}{\sin \Theta} \bar{P}_{n}^{|k|}(\cos \Theta) \hat{\Phi}\right]$

$\bar{P}_{n}^{|k|}(\cos \Theta)$ being the normalized associated Legendre functions as defined by Belousov in [14]. The modal expansion coefficients $A_{1,2 n k}$ in (20) can be determined from the knowledge of the tangential electric field on the scanning sphere:

$A_{1,2_{n k}}=\frac{1}{\beta g_{1,2 n}(\beta d)}$.

$\cdot \int_{0}^{\pi} \int_{-\pi}^{\pi} \underline{E}_{t}(d, \vartheta, \varphi) \cdot \underline{f}_{1,2_{n k}}^{*}(\vartheta) \mathrm{e}^{-\mathrm{j} k \varphi} \sin \vartheta \mathrm{d} \varphi \mathrm{d} \vartheta$

where $\left(^{*}\right)$ denotes the complex conjugate and

$g_{1_{n}}(x)=h_{n}^{(2)}(x)$

$g_{2_{n}}(x)=\frac{1}{x} \frac{\mathrm{d}}{\mathrm{d} x}\left[x h_{n}^{(2)}(x)\right]$

$h_{n}^{(2)}(x)$ being the spherical Hankel function of second kind and order $n$.

As shown in [11], the integration over $\varphi$ in relation (25) can be efficiently carried out by expanding the tangential electric field components in Fourier series with respect to $\varphi$, i.e.,
$E_{\vartheta, \varphi}(d, \vartheta, \varphi)=\sum_{m=-M_{\varphi}}^{M_{\varphi}} G_{\vartheta, \varphi m}(\vartheta) \mathrm{e}^{\mathrm{j} m \varphi}$

where $M_{\varphi}=\operatorname{Int}\left(\chi^{*} \beta a \sin \vartheta\right)+1, \quad \chi^{*}$ being the azimuthal excess bandwidth factor [11]. In a quite similar way, the remaining integration over $\vartheta$ can be efficiently performed by expanding the components of $\underline{G}_{k}(\vartheta)$ and $\underline{f}_{1,2 n k}(\vartheta)$ in Fourier series. Accordingly,

$A_{1,2_{n k}}=\frac{2 \pi}{\beta g_{1,2 n}(\beta d)}$.

$\cdot \sum_{\ell=-N_{\max }}^{N_{\max }} \sum_{i=-N_{\max }}^{N_{\max }} \underline{G}_{k \ell} \cdot \underline{f}_{1,2 n k i}^{*} \int_{0}^{\pi} \mathrm{e}^{\mathrm{j}(\ell-i) \vartheta} \sin \vartheta \mathrm{d} \vartheta$

It is worthy to note that, in order to evaluate the Fourier series coefficients of the components of $\underline{G}_{k}(\vartheta)$ and $\underline{f}_{1,2 n k}(\vartheta)$, it is necessary, as described in [11], to extend

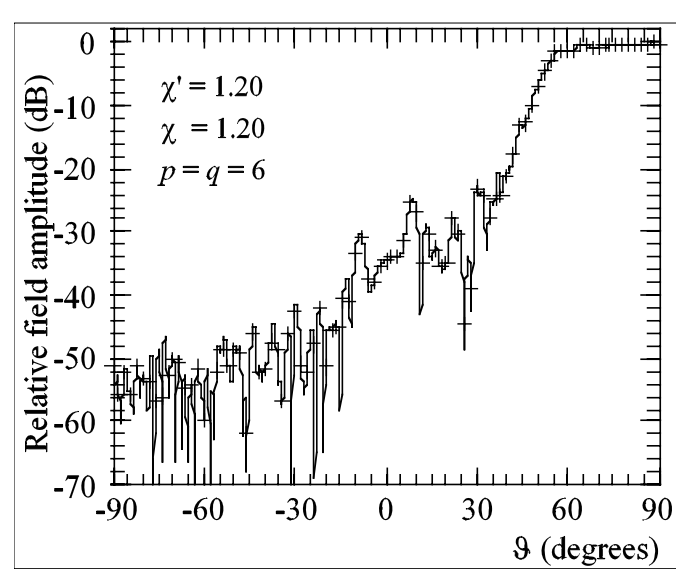

Fig. (6). Amplitude of the electric NF $\vartheta$-component on the meridian at $\varphi=90^{\circ}$. Solid line: exact. Crosses: interpolated.

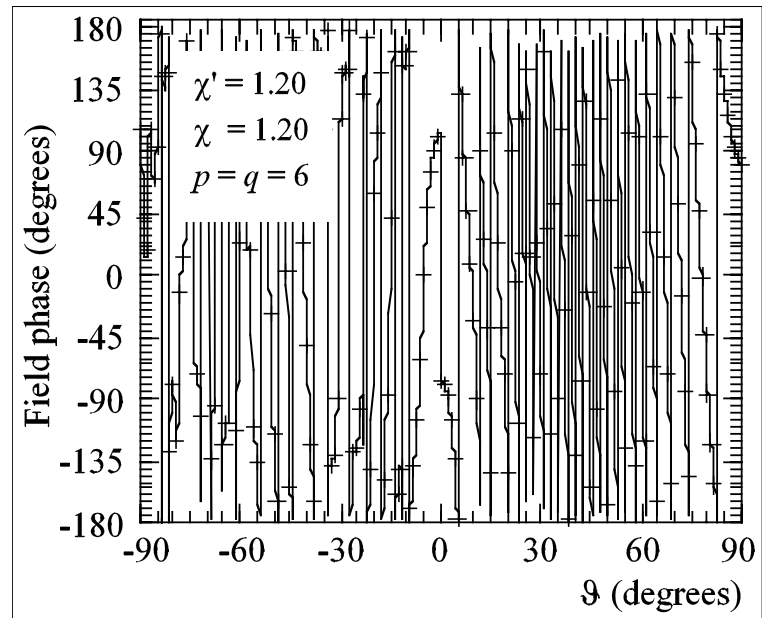

Fig. (7). Phase of the electric NF $\vartheta$-component on the meridian at $\varphi$ $=90^{\circ}$. Solid line: exact. Crosses: interpolated. 
these components from $[0, \pi]$ to the range $[-\pi, \pi]$. Moreover, to take advantage of the numerical efficiency of the standard FFT algorithm, the number of NF parallels and the number of samples on them must be the first power of two greater or equal to $N_{\max }$ and $2 M_{\varphi}$, respectively.

\section{NUMERICAL RESULTS}

Two sets of simulations are reported in the following. The former (from Figs. 6 to 13) refers to an elongated AUT, whereas, the latter (from Figs. 14 to 17) is relevant to the case of a quasi-planar antenna.

In the first case, the numerical tests refer to a spiral wrapping a sphere having radius $d=35 \lambda$ and to a uniform planar array of elementary Huygens sources polarized along the $z$ axis and spaced by $0.5 \lambda$. These sources cover an elliptical zone in the plane $y=0$, with major and minor semiaxes equal to $25 \lambda$ and $6 \lambda$, so that the antenna can be very well fitted by a prolate ellipsoid. Figs. (6 and 7) show the reconstruction of the amplitude and phase of the electric NF $\vartheta$-component on the meridian at $\varphi=90^{\circ}$. As can be seen, there is an excellent agreement between the exact field and the reconstructed one. It is useful to note that, in the zones of the spiral determined by the 60 samples around the poles, we have adopted an excess bandwidth factor such that the sample spacing is reduced exactly by a factor 9 . The accuracy in the NF interpolation is also confirmed by the values of the maximum and mean-square errors (normalized to the field maximum value on the sphere) reported in Figs. (8 and 9) for $p=q$ ranging from 3 to $9, \chi^{\prime}=1.20$ (save for the polar zones), and $\chi=1.10,1.15,1.20,1.25$. As expected, they decrease up to very low values on increasing the oversampling factor and/or the number of retained samples. The robustness of the algorithm has been investigated by adding random errors to the exact samples. These errors simulate a background noise (bounded to $\Delta a$ in amplitude and with arbitrary phase) and an uncertainty on the field samples of $\pm \Delta a_{r}$ in amplitude and $\pm \Delta \sigma$ in phase. As shown in Figs. (10 and 11), the interpolation algorithm works well also in presence of error affected data. The reconstructions of the an-

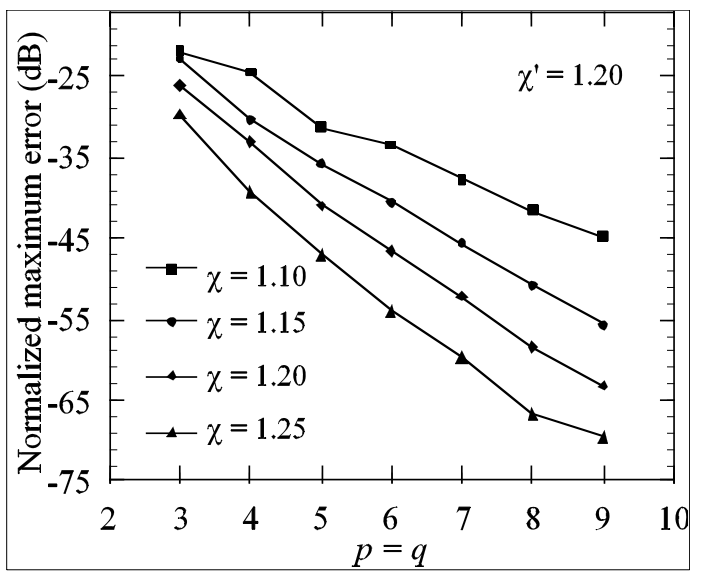

Fig. (8). Normalized maximum errors in the reconstruction of the electric NF $\vartheta$-component. tenna FF pattern in the principal planes are shown in Figs. (12 and 13). As can be seen, the reconstructions are very accurate. It is worthy to note that the number of samples on the spiral is 14852 , significantly less than that (66 940) required by the approach proposed in [9]. In particular, the number of "regular samples" at spacing $\Delta \xi$ is 12932 , whereas the number of "extra samples" at reduced spacing is 1920 . Moreover, the number of employed samples results to be much less than that ( 130562$)$ needed by the classical NFFF transformation with spherical scan [10].

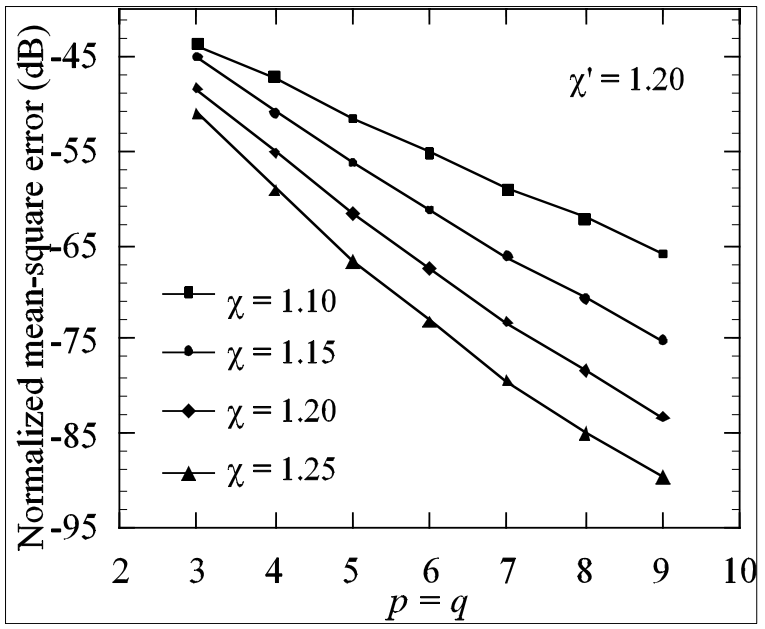

Fig. (9). Normalized mean-square errors in the reconstruction of the electric NF $\vartheta$-component.



Fig. (10). Amplitude of the electric NF $\vartheta$-component on the meridian at $\varphi=90^{\circ}$. Solid line: exact. Crosses: interpolated from error affected data.

The second set of figures refers to a spiral wrapping a sphere having radius $d=35 \lambda$ and to a uniform planar circular array with radius $19.8 \lambda$, lying in the plane $z=0$. Its elements (elementary Huygens sources linearly polarized along the $y$ axis) are radially and azimuthally spaced by $0.5 \lambda$. The considered array has been modelled as enclosed in an oblate ellipsoid having $a=20 \lambda$ and $b=3 \lambda$. An example of NF reconstruction relevant to the meridian at $\varphi=90^{\circ}$ is reported in Fig. (14). Again, a very accurate reconstruction results. Fig. (15) confirms the algorithm accuracy by show- 


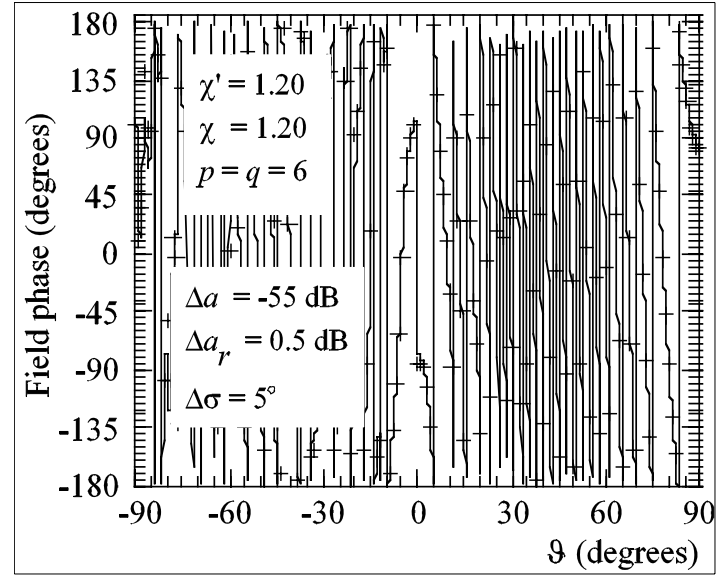

Fig. (11). Phase of the electric NF $\vartheta$-component on the meridian at $\varphi=90^{\circ}$. Solid line: exact. Crosses: interpolated from error affected data.

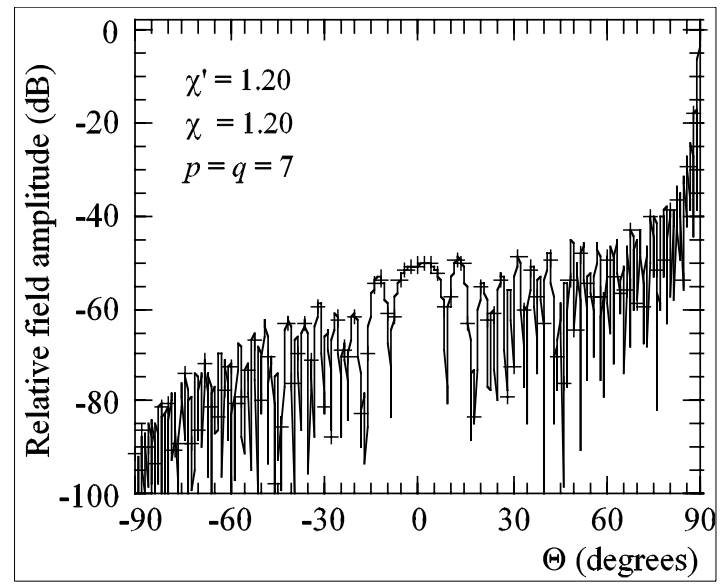

Fig. (12). E-plane pattern. Solid line: exact. Crosses: reconstructed from NF data.

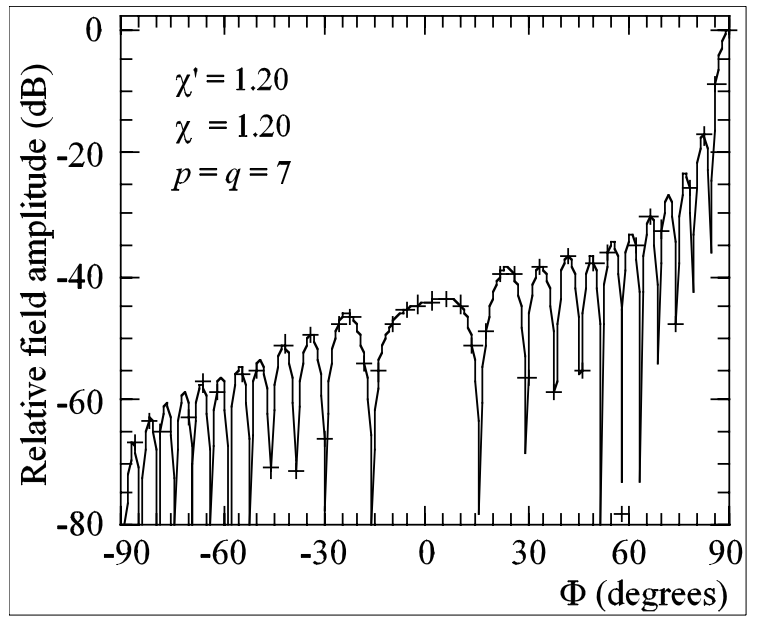

Fig. (13). H-plane pattern. Solid line: exact. Crosses: reconstructed from NF data.

ing the normalized maximum and mean-square errors for $p=q$ ranging from 3 to 10 and $\chi^{\prime}=\chi=1.20$. Note that, also in such a case, it has been necessary to increase $\chi^{\prime}$ when interpolating nearby the poles. In particular, we have adopted, in the zones of the spiral determined by the 20 samples around the poles, an excess bandwidth factor such that the sample spacing is reduced by a factor 13 . The reconstruction of the antenna FF pattern in the E plane is shown in Fig. (16). As can be seen, the exact and recovered fields are practically indistinguishable, thus assessing the effectiveness of the developed NF-FF transformation. Also in such a case, it can be interesting to compare the NF data required by the different techniques. The number of samples on the spiral is now 23763 , significantly less than that (42 700) required by the approach in [9]. In particular, the number of regular samples is 22803 , whereas the number of extra samples is 960 . Moreover, the number of used samples results to be much less than that (130 562) needed by the NF-FF transformation [10].

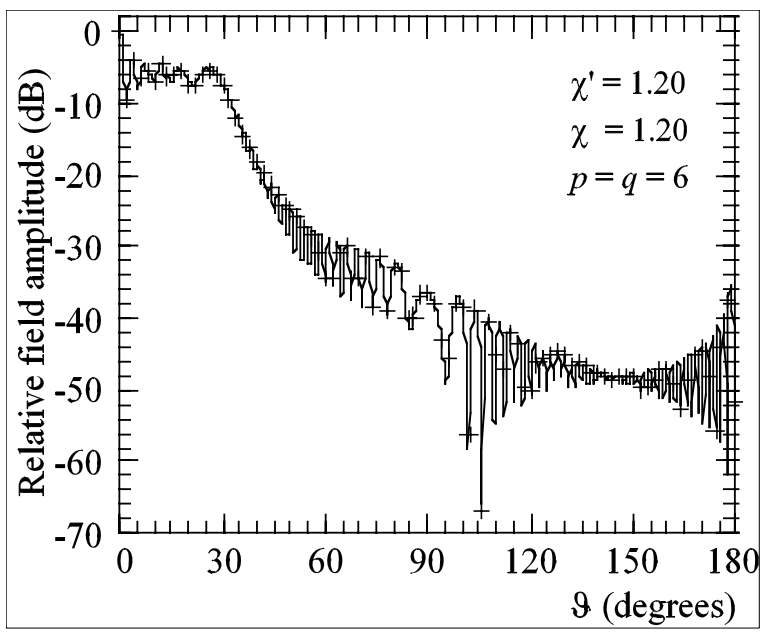

Fig. (14). Amplitude of the electric NF $\vartheta$-component on the meridian at $\varphi=90^{\circ}$. Solid line: exact. Crosses: interpolated.

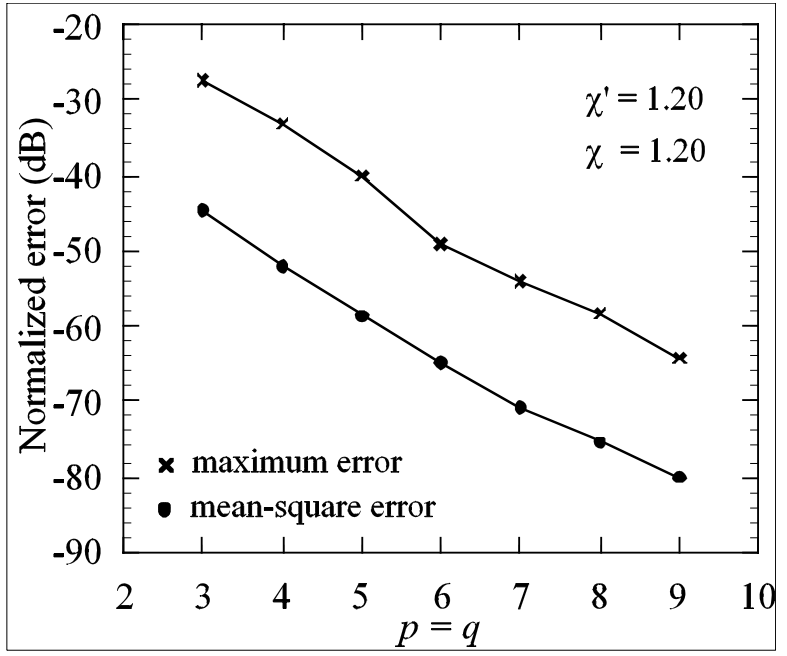

Fig. (15). Normalized errors in the reconstruction of the electric NF $\vartheta$-component.

A further example, which assesses the stability of the proposed NF-FF transformation technique, is reported in Fig. (17). It refers to the FF reconstruction obtained from error affected NF data. 


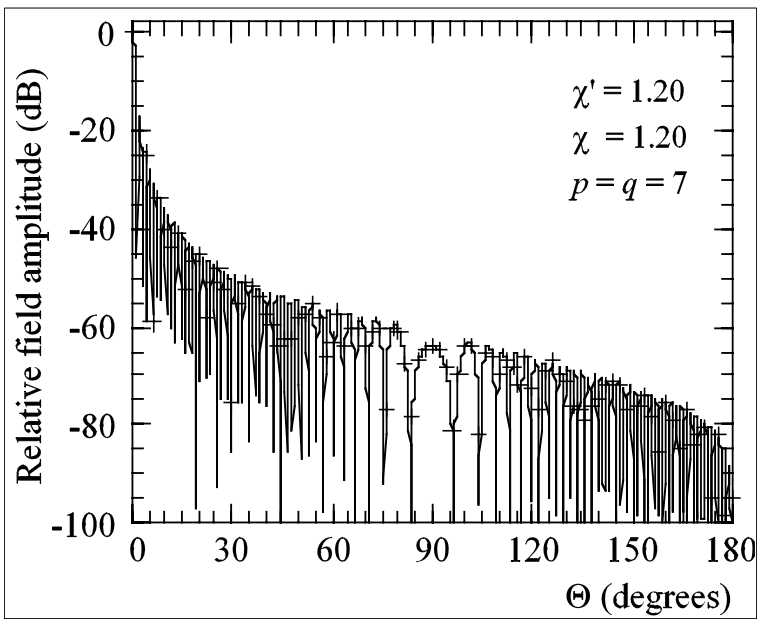

Fig. (16). E-plane pattern. Solid line: exact. Crosses: reconstructed from NF data.

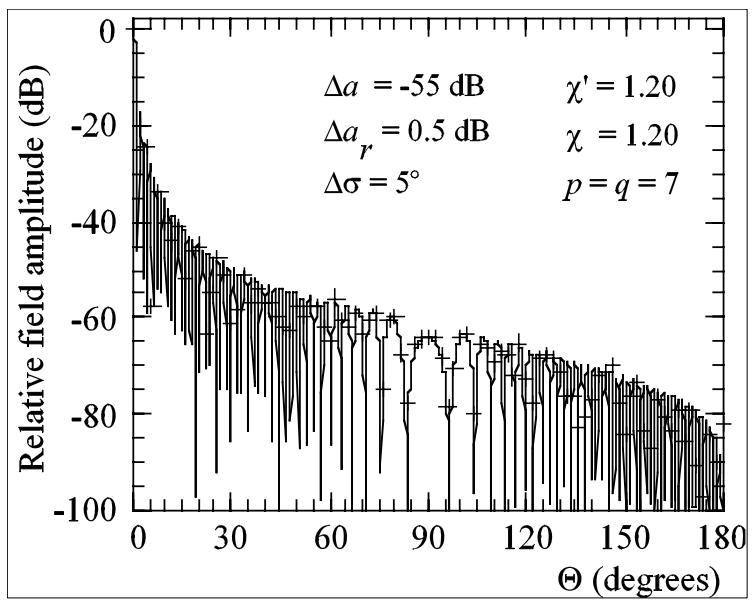

Fig. (17). E-plane pattern. Solid line: exact. Crosses: reconstructed from error affected NF data.

\section{CONCLUSIONS}

A fast, accurate and stable NF-FF transformation technique with spherical spiral scanning tailored for nonspherical antennas has been developed in this paper. To this end, an AUT with one or two predominant dimensions has been considered as enclosed in a prolate or oblate ellipsoid, respectively. By using these effective modellings, instead of the previously adopted spherical one, it is possible to remarkably reduce the number of the required NF data. Although, the optimal spiral, the corresponding phase factor and parameterization have been determined by applying a heuristic approach, the proposed techniques work very well as widely assessed by the numerical simulations.

\section{REFERENCES}

[1] "Special issue on near-field scanning techniques", IEEE Trans. Antennas Propagat., vol. AP-36, pp. 727-901, June 1988.

[2] A.D. Yaghjian, "An overview of near-field antenna measurements", IEEE Trans. Antennas Propagat., vol. AP-34, pp. 30-45, January 1986.

[3] C. Gennarelli, G. Riccio, F. D'Agostino, and F. Ferrara, "Near-field - far-field transformation techniques", vol. 1, CUES, Salerno, Italy, November 2004.

[4] R.G. Yaccarino, L.I. Williams, and Y. Rahmat-Samii, "Linear spiral sampling for the bipolar planar antenna measurement technique”, IEEE Trans. Antennas Propagat., vol. AP-44, pp. 10491051, July 1996.

[5] O.M. Bucci, C. Gennarelli, G.Riccio, and C. Savarese, "Nonredundant NF-FF transformation with helicoidal scanning", J. Electromagn. Waves Appl., vol. 15, pp. 1507-1519, 2001.

[6] O.M. Bucci, F. D’Agostino, C. Gennarelli, G. Riccio, and C. Savarese, "Probe compensated FF reconstruction by NF planar spiral scanning", IEE Proc. - Microw., Antennas Propagat., vol. 149, pp. 119-123, April 2002.

[7] O.M. Bucci, F. D’Agostino, C. Gennarelli, G. Riccio, and C. Savarese, "NF-FF transformation with spherical spiral scanning", IEEE Antennas Wireless Propagat. Lett., vol. 2, pp. 263-266, 2003.

[8] O.M. Bucci, C. Gennarelli, and C. Savarese, "Representation of electromagnetic fields over arbitrary surfaces by a finite and nonredundant number of samples", IEEE Trans. Antennas Propagat. vol. 46, pp. 351-359, 1998.

[9] F. D'Agostino, C. Gennarelli, G. Riccio, and C. Savarese, "Theoretical foundations of near-field-far-field transformations with spiral scannings", Prog. Electromagn. Res., PIER 61, pp. 193-214, 2006.

[10] J.E. Hansen, "Spherical near-field antenna measurements", IEE Electromagnetic Waves Series, London, Peter Peregrinus Ltd., UK, 1998.

[11] O.M. Bucci, F. D’Agostino, C. Gennarelli, G. Riccio, and C. Savarese, "Data reduction in the NF-FF transformation technique with spherical scanning", J. Electromagn. Waves Appl., vol.15, pp.755775, 2001

[12] O.M. Bucci, C. Gennarelli, G. Riccio, and C. Sa-varese, "Nearfield-far-field transformation from nonredundant plane-polar data: effective modellings of the source", IEE Proc. - Microw., Antennas Propagat., vol. 145, pp. 33-38, February 1998.

[13] O.M. Bucci, C. Gennarelli, G. Riccio, and C. Savarese, "NF-FF transformation with cylindrical scanning: an effective technique for elongated antennas", IEE Proc. - Microw., Antennas Propagat., vol. 145, pp. 369-374, October 1998.

[14] S.L. Belousov, "Tables of normalized associated Legendre polynomials", Pergamon Press, Oxford, 1962.

Received: October 27, 2008

(C) D'Agostino et al.; Licensee Bentham Open .

This is an open access article licensed under the terms of the Creative Commons Attribution Non-Commercial License (http://creativecommons.org/licenses/by-nc/3.0/) which permits unrestricted, non-commercial use, distribution and reproduction in any medium, provided the work is properly cited. 